\title{
Implementación de un modelo porcino de trasplante hepático en Chile
}

\author{
Gabriela Ochoa S. ${ }^{1}$, Carlo Marino C. ${ }^{1}$, Sergio Riveros G. ${ }^{1}$, Emilio Morales Z. ${ }^{1}$, \\ Cristian Jarry T. ${ }^{1}$, Macarena Viñuela M. ${ }^{1,}$ Leyla Alegría V. ${ }^{2}$, María Josefina Zenteno S. ${ }^{3}$, \\ Jorge Martínez C. ${ }^{1}$, Pablo Achurra T. ${ }^{1}$ y Rolando Rebolledo A. ${ }^{1,4}$
}

\section{Implementation of a porcine liver transplant model in Chile}

Objective: To Present the implementation of the first porcine model of liver transplant in Chile and its results. Materials and Methods: In the context of an investigation based on normothermic perfusion that was subsidized by the National Fund for Scientific and Technological Development, surgical and anesthetic protocols were implemented. Two pigs were selected by weight (35-40 kg), in which one was a donor and the other a receptor; submitted to procure and as a transplant recipient, respectively. The analysis was executed with descriptive statistics. Results: 26 experiments (June 2018-October 2019) were performed. The protocol includes 7 stages: Preparation, Instrumentalization, Procure or Hepatectomy, Anhepatic Time, Phase of Ischemia-Reperfusion, Monitoring and Euthanasia. The first three stages are similar in both pigs; meanwhile the last four are exclusive to the receptor. $92.3 \%(24 / 26)$ survived the surgery and $76.9 \%(20 / 26)$ survived the follow up, including the pilots. Average surgical time was 170 minutes, and the anhepatic time was 33 minutes. The mean arterial pressure decreased after the reperfusion and it improved towards the end of the monitorization $(67,4 \mathrm{mmHg}$ average) In the follow up, pigs presented acidosis (7.21 average). Vasoactive drugs were necessary in 12 experiments. Discussion: We managed to implement a simple and reproducible model of porcine liver transplant without extracorporeal circulation or venovenous by-pass. This was possible due to the standardization of surgical technique and perioperative cares. Conclusions: The consolidation of porcine liver transplant model is the first step in pre-clinical research for the development of new technologies in liver transplant in Chile.

Key words: liver transplantation; experimental surgery.

\section{Resumen}

Objetivo: Presentar la implementación del primer modelo porcino de trasplante hepático (TH) en Chile y sus resultados. Material y Método: Se implementó un protocolo quirúrgico y anestésico en el contexto de una investigación en perfusión normotérmica hepática financiada por un Fondo Nacional de Desarrollo Científico y Tecnológico. Los cerdos fueron seleccionados por peso (35-40 kilos), en cada experimento se utilizó dos, donante y receptor, sometidos a procura y trasplante respectivamente. El análisis se realizó con estadística descriptiva. Resultados: Se realizaron 26 experimentos (marzo de 2018-octubre de 2019). El protocolo consta de 7 etapas: Preparación, Instrumentalización, Procura o Hepatectomía, Tiempo Anhepático, Etapa de Isquemia-Reperfusión, Monitorización y Eutanasia. Las primeras tres son similares en ambos cerdos, y desde la cuarta en adelante corresponde sólo al receptor. La supervivencia a la cirugía fue de $92,3 \%$ (24/26) y al seguimiento de $76,9 \%$ (20/26). La mortalidad se produjo por inestabilidad cardiovascular postreperfusión portal. El tiempo quirúrgico promedio fue de $170 \mathrm{~min}$, y el tiempo anhepático de 33 min. La PAM cursó una baja postreperfusión con recuperación al final de la monitorización (67,4 mmHg media) y la tendencia en ph fue a acidosis al final del seguimiento (7,21 media). Se requirió drogas vasoactivas en 12 casos. Discusión: Logramos implementar un modelo experimental simplificado y reproducible de TH sin necesidad de circulación extracorpórea ni puente veno-venoso gracias a la estandarización de la técnica quirúrgica y de los cuidados perioperatorios. Conclusiones: La consolidación de un modelo experimental significa el primer paso en investigación preclínica de nuevas tecnologías asociadas al TH en Chile.

Palabras clave: trasplante hepático porcino; cirugía experimental.
'Departamento de Cirugía Digestiva. Escuela de Medicina. Facultad de Medicina. Pontificia Universidad Católica de Chile. Santiago, Chile.

${ }^{2}$ Departamento de

Medicina Intensiva. Escuela de Medicina. Facultad de Medicina. Pontificia Universidad Católica de Chile. Santiago, Chile. ${ }^{3}$ Centro de Habilidades Quirúrgicas. Departamento de Cirugía Oriente. Facultad de Medicina. Universidad de Chile. Santiago, Chile. ${ }^{4}$ Instituto de Ingeniería Biológica y Médica. Pontificia Universidad Católica de Chile. Santiago, Chile.

Recibido el 4 de noviembre de 2019 y aceptado para publicación el 9 de marzo de 2020 .

Correspondencia a: Rolando Rebolledo A. rareboll@uc.cl 


\section{Introducción}

El trasplante hepático $(\mathrm{TH})$ es la única opción curativa para los pacientes con cirrosis hepática avanzada, falla hepática fulminante y neoplasias primarias y secundarias del hígado en el marco de criterios estrictos previamente publicados ${ }^{1}$. La escasez de donantes, el continuo crecimiento de las listas de espera en Chile y el mundo, y la alta tasa de mortalidad en la lista, generan la constante necesidad de desarrollar nuevas técnicas y tecnologías en TH. Esto sólo se logra a través de los esfuerzos constantes en investigación clínica y básica aplicada ${ }^{2}$.

El desarrollo de la técnica de TH ha estado unido desde el inicio al conocimiento aportado por los modelos experimentales en animales ${ }^{2}$. Los primeros estudios fueron realizados en perros por Godric en $1956^{3}$. Al poco andar, en 1959, se describió el primer modelo en $\operatorname{cerdos}^{4}$, y desde entonces múltiples grupos han publicado sus experiencias y resulta$\operatorname{dos}^{7-9}$. Los cerdos tienen una gran similitud anatómica, fisiológica y de tamaño con los humanos ${ }^{10}$, lo cual permite generar estudios traslacionales fidedignos. Esto, sumado al desarrollo de los cuidados perioperatorios y el perfeccionamiento de la técnica quirúrgica permite desarrollar nuevas estrategias terapéuticas en $\mathrm{TH}^{11-13}$. Sin embargo, muchos de los modelos descritos no son reproducibles por su complejidad, que en la mayoría de los casos incluye uso de puente veno-venoso, y alta mortalidad peri operatoria. El modelo simplificado de Fondevilla ha logrado resultados exitosos sin necesidad de un puente veno-venoso, consiguiendo la estabilidad hemodinámica a través de minimizar el tiempo anhepático en el receptor ${ }^{12}$.

Nuestro grupo ha desarrollado una línea de investigación en perfusión normotérmica hepática, para lo cual fue necesario desarrollar un modelo experimental de trasplante. En este contexto, hicimos una adaptación del modelo publicado por Fondevilla, modificando algunos aspectos de la técnica quirúrgica, optimizando el manejo anestésico y los cuidados postoperatorio ${ }^{14,15}$. El objetivo de este artículo es presentar la implementación del primer modelo experimental porcino de $\mathrm{TH}$ descrito en Chile y sus resultados.

\section{Material y Método}

Se realizó un protocolo del procedimiento consistente en siete etapas, en las cuales se aborda los aspectos principales de anestesia, técnica quirúrgica y cuidados perioperatorios. Las primeras tres etapas son comunes al donante y receptor, y desde la cuarta en adelante corresponde únicamente al receptor.

Los cerdos fueron seleccionados por peso en un rango de 35 a $40 \mathrm{~kg}$. El grupo sanguíneo y pruebas cruzadas no fueron determinados.

El financiamiento fue obtenido a través de un Fondo Nacional de Desarrollo Científico y Tecnológico (FONDECYT No 11160928), destinado a la investigación en perfusión hepática, y en forma paralela se desarrolló el modelo de TH porcino. Por lo mismo, los cerdos fueron aleatorizados en 4 grupos de estudio: control frío, control máquina, isquémico frío e isquémico máquina. La categorización isquémico corresponde a experimentos en que se obtiene un injerto hipoperfundido a través de la extracción del $30 \%$ de la volemia del donante $(55 \mathrm{cc} / \mathrm{kg})$. La categorización frío o máquina corresponde al tipo de preservación realizado.

El proyecto fue aprobado por el Comité de Ética de Bienestar Animal de la Pontificia Universidad Católica de Chile con el acta de aprobación $\mathrm{n}^{\circ}$ 160126009 y cumple con normativa establecida por la comisión europea para investigación con animales.

Los resultados se analizaron con estadística descriptiva.

\section{Resultados}

Se realizaron 26 procedimientos entre marzo de 2018 y octubre de 2019 . Todos dentro del proyecto de perfusión normotérmica hepática, por lo cual fueron aleatorizados según el protocolo de investigación. Hubo 5 modelos pilotos que fueron incluidos en los resultados.

\section{Descripción del procedimiento}

\section{$1^{\circ}$ Preparación}

Los cerdos se mantienen en ayuno a sólidos $8 \mathrm{~h}$ y a líquidos $2 \mathrm{~h}$. Se premedican en el corral con midazolam $0,5 \mathrm{mg} / \mathrm{kg}$ más ketamina $25 \mathrm{mg} /$ $\mathrm{kg}$ im. Posteriormente al derribo son trasladados al pabellón. La inducción anestésica contempla preoxigenación, instalación de vía venosa periférica en la vena dorsal del pabellón auricular, monitorización cardiopulmonar no invasiva, analgesia con fentanilo $4 \mathrm{ug} / \mathrm{kg}$, relajación muscular con atracurio $0,5 \mathrm{mg} / \mathrm{kg}$, propofol en bolo $1 \mathrm{mg} / \mathrm{kg}$ e intubación orotraqueal. Se administra una analgesia multimodal protegiendo de este modo la integridad nociceptiva de los ejemplares, basada en opioides y lidocaína. 


\section{$2^{\circ}$ Instrumentalización}

Se inicia la ventilación mecánica monitorizada por capnografía.

El tiempo quirúrgico se inicia con el abordaje cervical para la canulación de la vena yugular externa para acceso venoso central y la arteria carótida común para presión arterial invasiva.

\section{$3^{\circ}$ Procuramiento - donante}

La mantención de la anestesia contempla: analgesia con fentanilo en bolos según requerimientos, relajación muscular con atracurio $0,5 \mathrm{mg} / \mathrm{kg} /$ hora en infusión continua y volemización con suero ringer lactato a $10 \mathrm{cc} / \mathrm{kg} / \mathrm{h}$.

El tiempo quirúrgico continúa con el abordaje abdominal por laparotomía xifopubiana, guiándose por la línea intermamaria y resguardando no lesionar la uretra en el caso de los machos (Figura 1). Se realiza la evisceración de asas intestinales hacia la izquierda exponiendo el retroperitoneo (Figura 2). En el cerdo la vena cava inferior (VCI) se encuentra incluida en el hígado, por lo que se libera desde su segmento infrahepático (VCII) hasta el segmento suprahepático (VCIS) (Figura 3), quedando así libre del retroperitoneo. El segmento VCII se debe dejar identificado con lazos de seda no oclusivos.

Se continúa con la liberación del omento menor y la disección del hilio hepático de izquierda a derecha, cercano a la curvatura menor avanzando hacia el borde duodenal. Se procede a la identificación de la arteria hepática común (AHC) teniendo extremo cuidado de no traumatizarla para evitar complica-

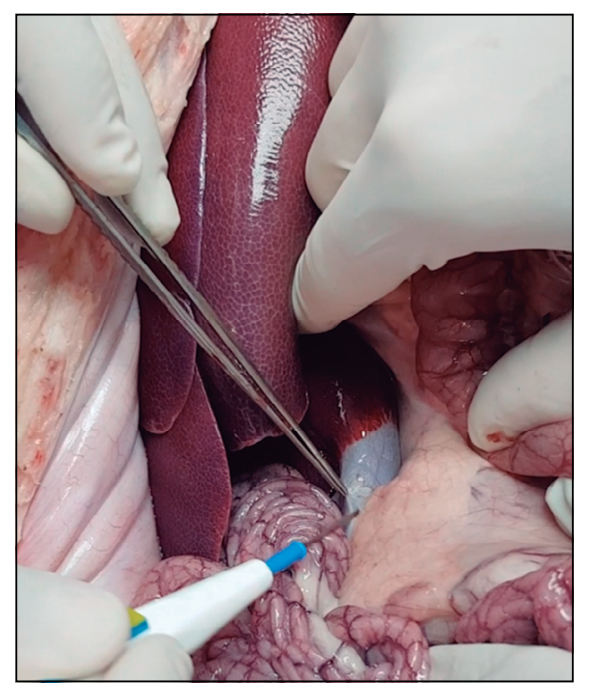

Figura 2. Abordaje abdominal. Exposición de retroperitoneo para abordaje de VCII. ciones por daño mural. Es necesario respetar todo su trayecto hasta la aorta. Se identifica y secciona la rama correspondiente a la arteria gástrica izquierda. A continuación, se libera la vena porta (VP) caudal al paso de AHC, siendo habitual encontrar múltiples adenopatías que es necesario disecar con adecuada hemostasia de los pedículos ganglionares

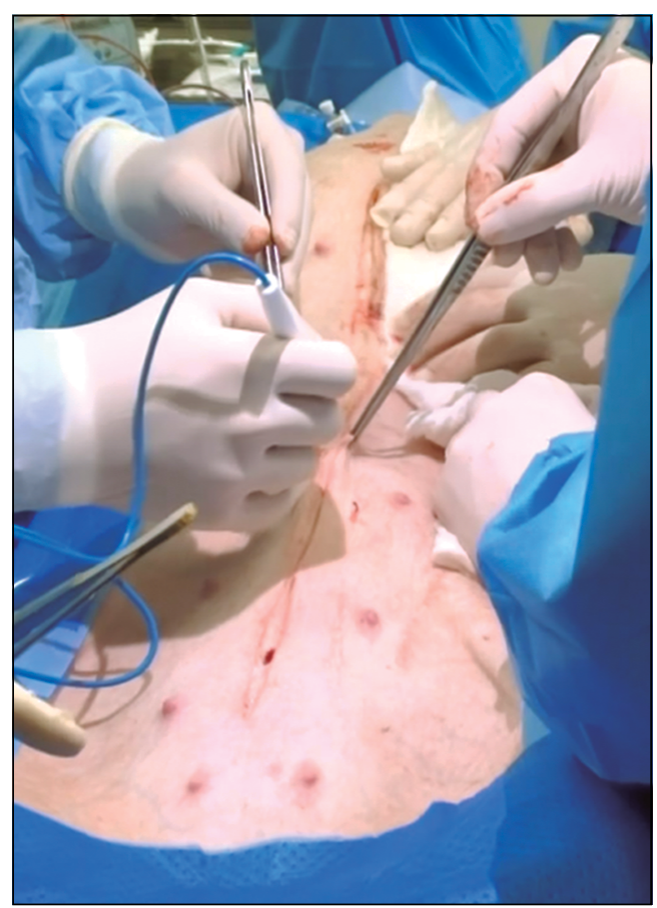

Figura 1. Abordaje abdominal. Laparotomía xifopubiana.

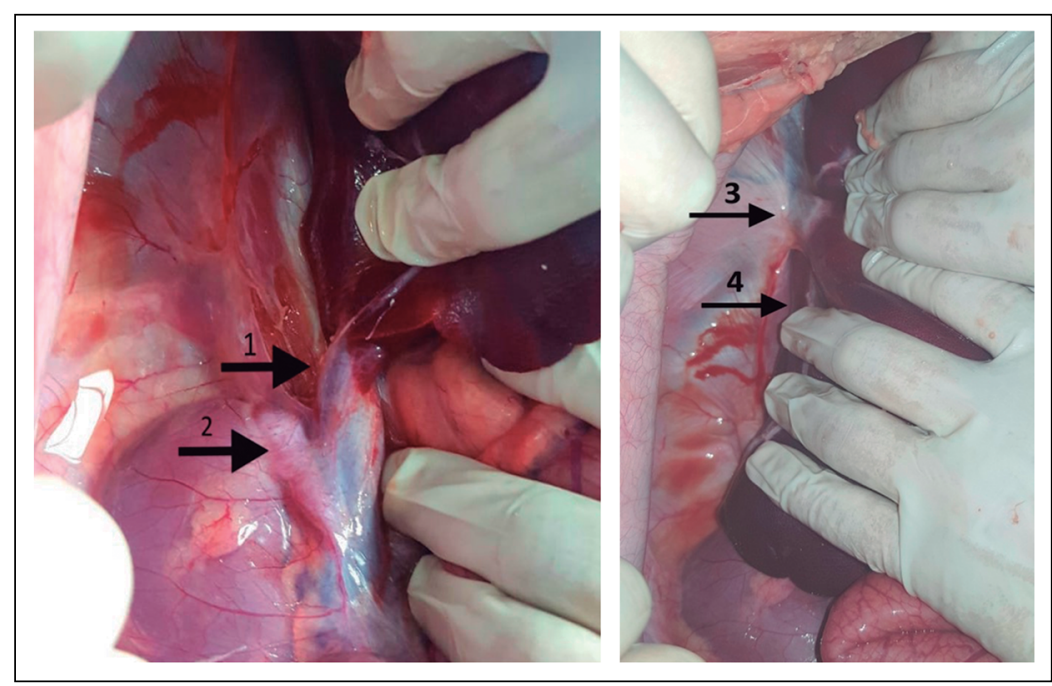

Figura 3. Vena cava inferior. 1) Exposición de VCIl; 2) Vena renal derecha; 3) VCIS; 4) Trayecto intrahepático de VCI. 
Figura 4. Disección de hilio hepático en el receptor. 1) $A H C$ (seccionada en explante); 2) Vena porta; 3) Colédoco (seccionado en explante) y abordaje de aorta abdominal distal en el donante; 4) Aorta; 5) $A H C$; 6) Arteria mesentérica superior; 7) $\mathrm{VCl}$.

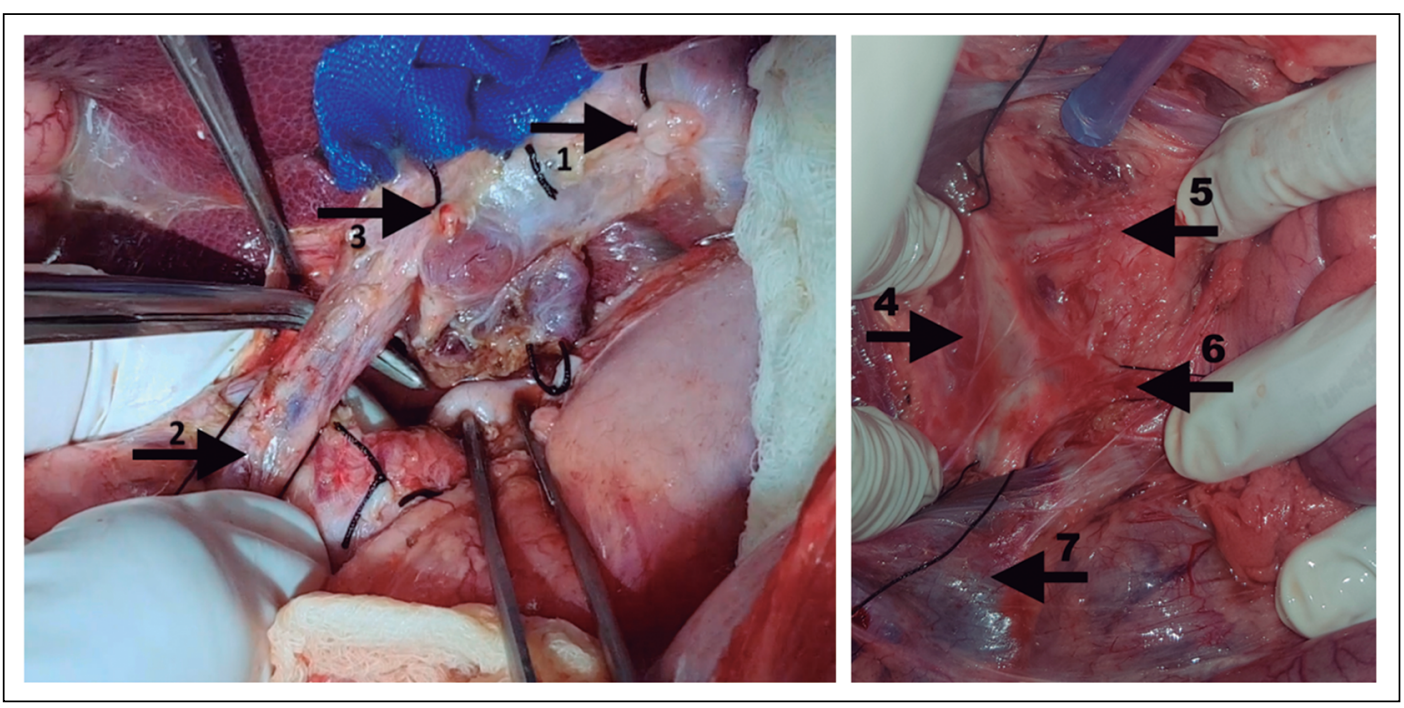

(Figura 4). Se deja un lazo elástico y de seda 2,0 sin traccionar, teniendo cuidado de no ocluir el flujo. Luego se realiza la sección del colédoco cercano al borde duodenal sin esqueletizarlo para no provocar isquemia. Se procede a lavar la vía biliar con suero fisiológico en forma retrógrada y anterógrada para evitar la precipitación de las sales biliares en la preservación en frío.

Se individualiza la aorta cercana a la bifurcación iliaca asegurando un buen paso por posterior dejando dos lazos simples de seda 2.0. Se debe rechazar el páncreas y duodeno hacía izquierda (Kocher-like) e identificar y lazar la arteria mesentérica superior. Cefálico a ésta se ubica la AHC de trayecto retroportal (Figura 4). Se realiza el paso superior de la aorta 1-2 cm sobre la emergencia de la AHC, dejando un lazo de seda y espacio suficiente para colocar clamp. Una vez identificado y aislados todos los elementos vasculares principales, se administra heparina de bajo peso molecular $300 \mathrm{UI} / \mathrm{kg}$ iv y se preparan 1 litro de solución de preservación (Custo$\operatorname{diol}^{\circledR}$ ) a $4{ }^{\circ} \mathrm{C}$ usando bajadas de suero para canular directamente la aorta y la vena porta en ese orden. Una vez que se ha cumplido el tiempo de heparina, se debe cerrar la ligadura de la arteria mesentérica superior, cerrar la ligadura aórtica distal y colocar un clamp aórtico proximal. Se abre el diafragma para exposición de la VCIS y se inicia la perfusión con Custodiol ${ }^{\circledR}$. Simultáneamente y dejando pinzas vasculares, se seccionan con tijera la VCIS, VCII y VP cuidando dejar el mayor largo posible. La aorta se secciona cefálico a la ligadura superior traccionándola y liberándola de las ramas lumbares hasta al menos $2 \mathrm{~cm}$ proximal a la emergencia de la $\mathrm{AHC}$, que debe ser vigilada para asegurar su indemnidad. El hígado liberado es destinado al tipo de preservación que corresponda según aleatorización por $3 \mathrm{~h}$. Se administra solución de eutanasia (tiopental $500 \mathrm{mg} \mathrm{ev}+\mathrm{T} 61)$.

\section{$3^{\circ}$ Hepatectomía - receptor}

El receptor es volemizado de manera diferente, para que pueda resistir la etapa anhepática. Inicialmente se administra 1.000 cc SF periférico, $500 \mathrm{cc}$ de Ringer lactato central y 500 cc albúmina al 5\% central, más ácido tranexámico $5 \mathrm{mg} / \mathrm{kg}$ ev lento como fibrinolítico.

La hepatectomía en el receptor es similar a la procura del donante en cuanto al abordaje de los elementos vasculares principales, pero su extensión y sección es diferente. Se inicia con la VCII, luego la VCIS donde es necesario ligar ambas venas frénicas ya que pueden producir complicaciones hemorrágicas al ser afluentes ésta, luego la VP y finalmente la AHC.

Una vez identificados los elementos vasculares principales, se procede a realizar una prueba de clampeo ocluyendo el flujo de la VCIS (Figura 5) con el objetivo de evaluar la respuesta del animal a la interrupción del retorno venoso y según su repercusión, volemizar generosamente a modo de preparación para el tiempo anhepático. Posteriormente, se procede a clampear y seccionar las estructuras vasculares lo más cercano al borde hepático: AHC, VCII, VP y, finalmente, VCIS. En este momento se inicia el tiempo anhepático (Figura 6). 


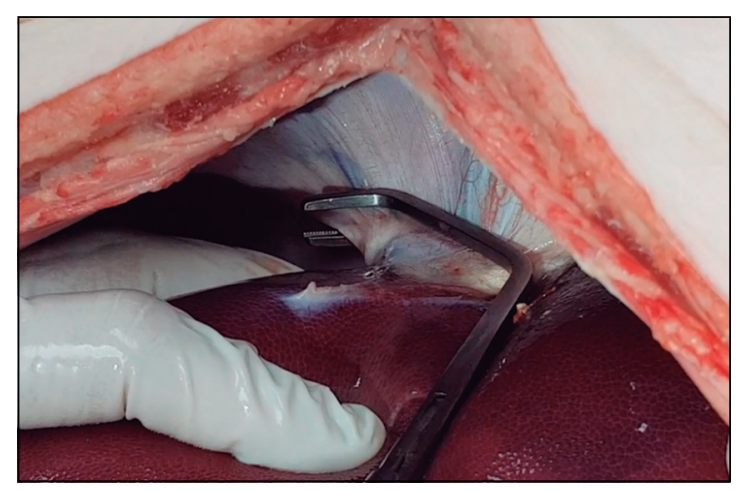

Figura 5. Test de clampeo VCIS en el receptor.

\section{$4^{\circ}$ Tiempo anhepático}

De acuerdo a la repercusión hemodinámica, se inicia apoyo con drogas vasoactivas (DVA) tituladas según presión arterial invasiva (epinefrina $0,01-0,1 \mathrm{ug} / \mathrm{kg} / \mathrm{min}$, efedrina 0,05 a $0,5 \mathrm{mg} / \mathrm{kg}$ en bolos, norepinefrina $0,01-0,1 \mathrm{ug} / \mathrm{kg} / \mathrm{min}$ ). El apoyo cardiovascular se realiza con cloruro de calcio $10 \%$ $10-15 \mathrm{mg} / \mathrm{kg}$ ev, sulfato de magnesio $25 \% 6 \mathrm{ml}$ ev y lidocaína $2 \% 4 \mathrm{ml} \mathrm{ev}$.

En este tiempo se realizan las anastomosis receptor-injerto (Figura 7). Primero la anastomosis de la VCIS con prolene 4.0 corrido y a continuación la anastomosis portal con prolene 5.0 corrido, en la cual se debe tomar la precaución de dejar factor de crecimiento. La liberación de los clamps parte con el portal para permitir un mínimo de lavado retrógrado que se exterioriza por la VCII desde el injerto, luego se clampea esta última vena y se abre el clamp de la VCIS. Posteriormente, se realiza la anastomosis de la VCII con prolene 4.0 corrido y la liberación de su clamp. Finalmente, se procede a la anastomosis de AHC con prolene 6.0 o 7.0 corrido y con la liberación de su clamp se logra reperfusión completa del hígado (Figura 8). Es necesario realizar una evaluación rigurosa de las anastomosis, cualquier sangrado mayor puede descompensar hemodinámicamente al animal, ya que el cerdo se caracteriza por tener una menor volemia. Es necesario tener resguardo con el retorno venoso mesentérico, evitando torsiones del intestino.

\section{$5^{\circ}$ Etapa isquemia - reperfusión}

En esta etapa el animal presenta la mayor inestabilidad. Generalmente se debe continuar o iniciar el apoyo con DVA y volumen. Se utiliza fenilefrina en bolos y se repite la dosis de ácido tranexámico. Además, se realiza las correcciones de hiperkalemia y ácido base según sea necesario (bicarbonato $2 / 3$ $1 \mathrm{mEq} / \mathrm{kg}$ ).

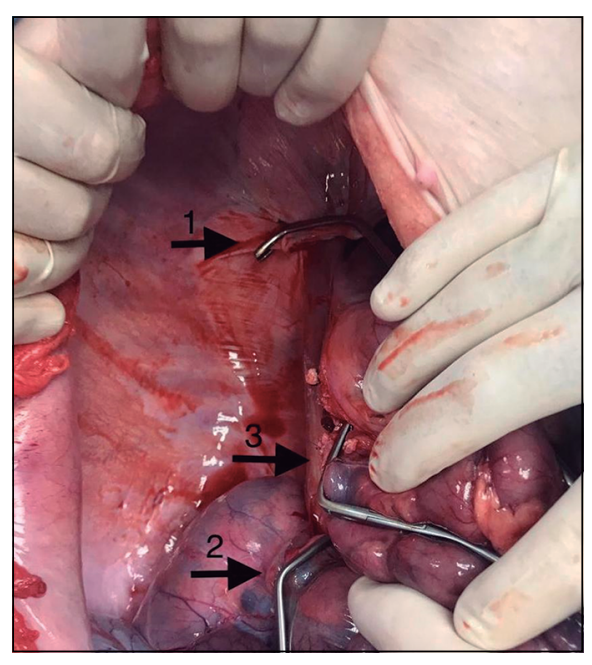

Figura 6. Tiempo anhepático en e receptor. 1) VCIS seccionada; 2) VCII seccionada; 3) VP seccionada.

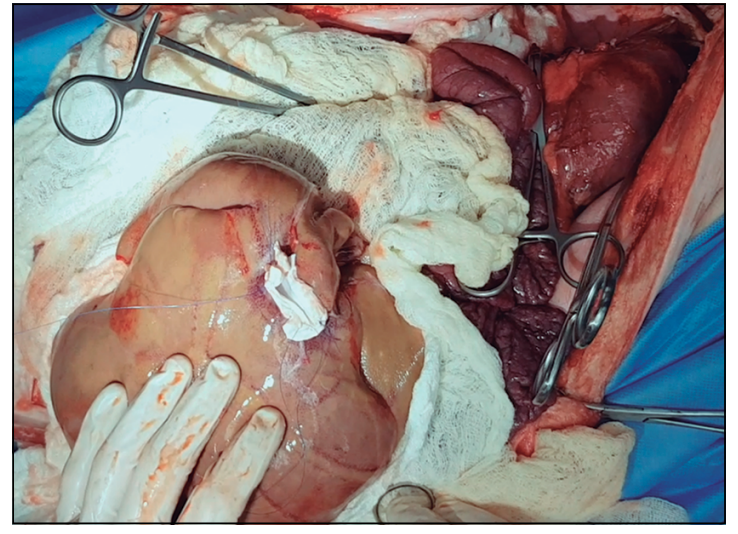

Figura 7. Visión de injerto y receptor en tiempo anhepático.
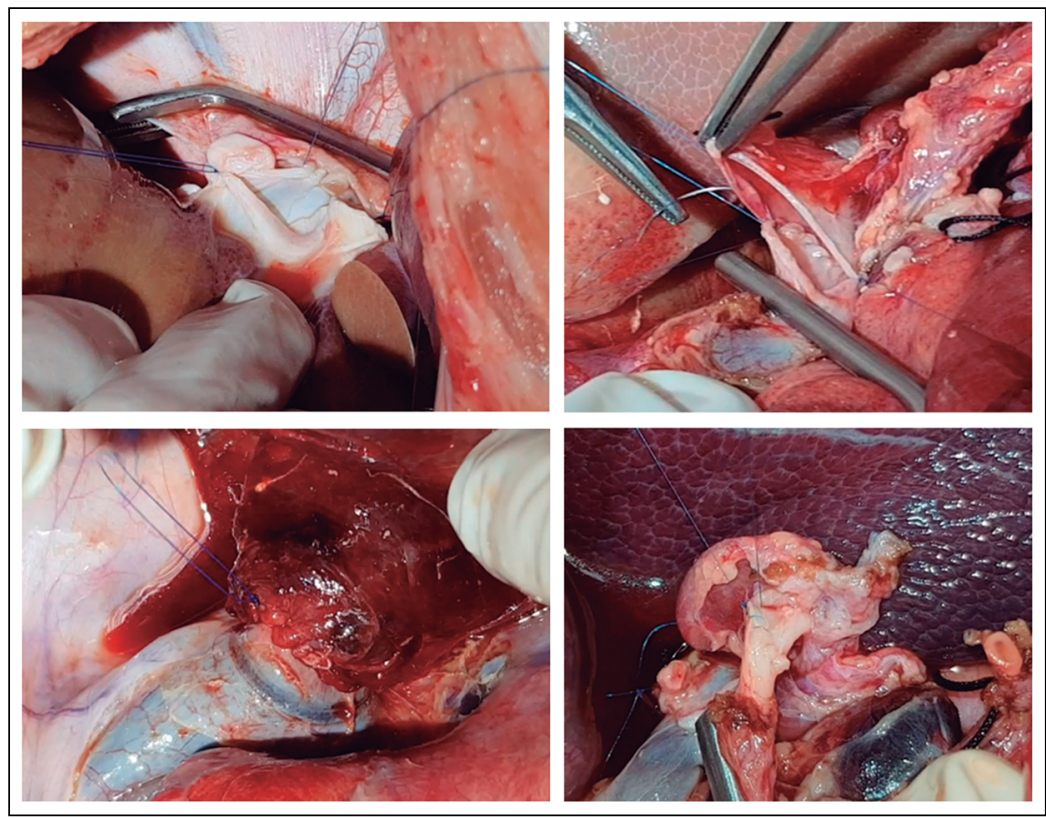

Figura 8. Anastomosis. 1) Anastomosis $\mathrm{VCSI}$; 2) Anastomosis $\mathrm{VP}$; 3) Anastomosis $\mathrm{VCll}$ 4) Anastomosis AHC. 
Tabla 1. Resumen de resultados en receptores

\begin{tabular}{|llc|}
\hline & & Media \\
\hline Sobrevivencia a procedimiento & $\%(\mathrm{n})$ & $92,3(24)$ \\
Filtración de anastomosis & $\%(\mathrm{n})$ & $76,5(20)$ \\
Trombosis vascular & $\%(\mathrm{n})$ & 0 \\
Tiempo quirúrgico total & $\%(\mathrm{n})$ & 0 \\
Tiempo anhepático & $\min (\mathrm{ds})$ & $170(42)$ \\
Tiempo hasta reperfusión arterial & $\min (\mathrm{ds})$ & $33(6)$ \\
PAM final de seguimiento & $\min (\mathrm{ds})$ & $92(18)$ \\
pH fin de seguimiento & $\mathrm{mmhg}(\mathrm{ds})$ & $67,4(12,8)$ \\
\hline
\end{tabular}

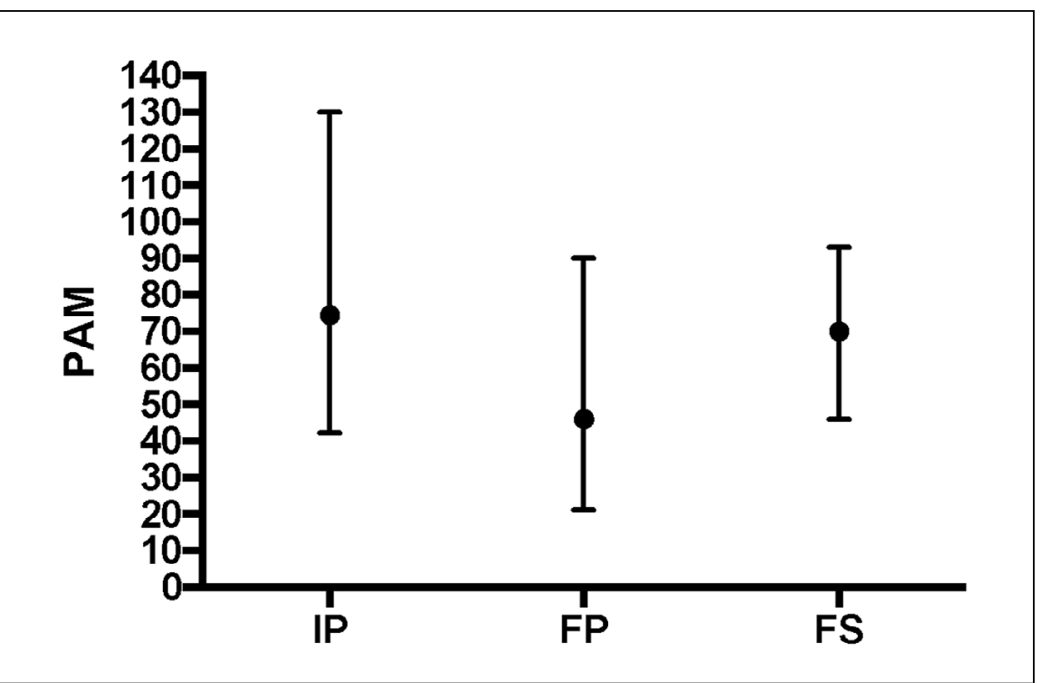

Figura 9. Gráfico de comportamiento PAM (media y rango) durante procedimiento en el receptor. IP inicio de procedimiento, FP fin del procedimiento y FS fin de seguimiento.

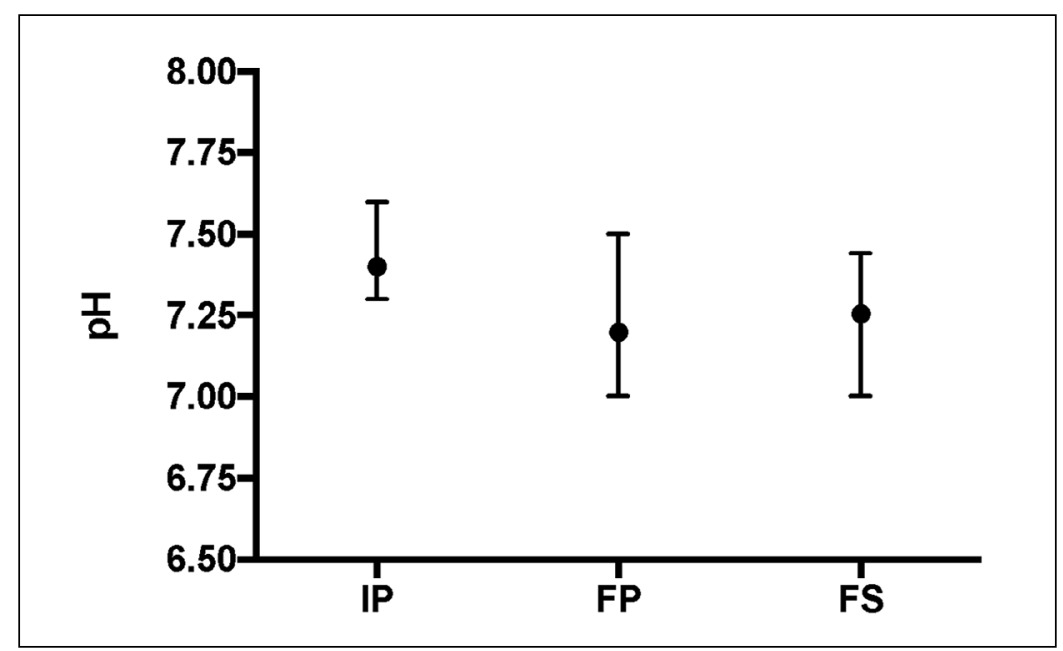

Figura 10. Gráfico de comportamiento $\mathrm{pH}$ (media y rango) durante procedimiento en el receptor. IP inicio de procedimiento, FP fin de procedimiento y FS fin de seguimiento.
Por último, se confecciona la anastomosis de la vía biliar con prolene 4.0 sobre una sonda Kehr exteriorizada por contrabertura y se cierra la laparotomía.

\section{$6^{\circ}$ Etapa UCI - monitorización}

Contempla $5 \mathrm{~h}$ desde la reperfusión portal. Se monitoriza con ECG, oximetría, capnografía, presión arterial invasiva, gases arteriales, glicemia, lactato, temperatura, medición de producción de orina y bilis.

La mantención de la anestesia es con TIVA, que se inicia $30 \mathrm{~min}$ antes de la reperfusión (ketamina, midazolam y fentanilo) a $0,5 \mathrm{ml} / \mathrm{kg} / \mathrm{h}$. Se debe considerar la acumulación de drogas según las horas administradas, por lo que se disminuye el aporte en forma escalonada.

\section{$7^{\circ}$ Eutanasia}

Terminada la monitorización, se reexplora el receptor poniendo énfasis en la presencia de sangre o bilis e indemnidad de las anastomosis. Se toma una biopsia de finalización y se administra solución de eutanasia.

La supervivencia a la cirugía fue de un 92,3\% (24/26), y la supervivencia al seguimiento postoperatorio de $5 \mathrm{~h}$ fue de $76,9 \%$ (20/26). El fallecimiento de los cerdos previo al término de la cirugía se debió a inestabilidad cardiovascular postreperfusión portal, al igual que los que fallecieron antes de completar el seguimiento. En el último caso se debió suspender el experimento por alteración persistente de parámetros ventilatorios y lactato.

El tiempo quirúrgico promedio, comprendido desde la cervicotomía hasta el cierre de la laparotomía, fue de $170 \mathrm{~min}$, y el tiempo anhepático promedio, transcurrido desde el clampeo de la VCIS hasta la reperfusión portal, fue de $33 \mathrm{~min}$.

La presión arterial media (PAM) promedio al inicio de la cirugía fue de $76,5 \mathrm{mmHg}$, cursando con una baja postreperfusión hasta $51,3 \mathrm{mmHg}$ y recuperación al final de la monitorización 67,4 mmHg (Figura 9).

El equilibrio ácido-base monitorizado a través de la medición de ph sanguíneo se comportó de manera similar, con un basal de 7,42 (media) a una baja post reperfusión 7,19 (media) y ascenso al final del seguimiento manteniendo una tendencia a la acidosis con un ph promedio final de 7,21 (Figura 10).

Fue necesario el uso de drogas vasoactivas epinefrina y norepinefrina en 12 de los experimentos durante la monitorización postoperatoria, todos con baja de los requerimientos al finalizar la cirugía. 


\section{Discusión}

Nuestro grupo logró implementar un modelo simplificado y reproducible de TH porcino sin necesidad de circulación extracorpórea ni puente veno-venoso gracias a la estandarización de la técnica quirúrgica y de los cuidados perioperatorios.

Los cerdos presentan la particularidad de tener el trayecto hepático de la VCI integrada al parénquima hepático, por lo cual en ellos no es posible realizar la técnica "piggy back", es decir, requiere una técnica sin preservación de cava ${ }^{10}$. Esto genera una gran repercusión hemodinámica durante la fase anhepática, al verse interrumpido de forma tan severa el retorno venoso ${ }^{15}$. Esta dificultad se suma a la imposibilidad de transfundir hemoderivados y factores de coagulación. En otros modelos, esta problemática se sortea a través de un puente veno-venoso, sin embargo, esto no está exento de complicaciones $\mathrm{y}$ añade complejidad y costo al procedimiento ${ }^{12}$. En nuestro modelo, se logra reducir este impacto gracias al conseguir tiempos anhepáticos breves, similares a otros grupos (promedio 20-30 min) y al manejo anestésico intensivo ${ }^{12,13,18}$. Fue fundamental la monitorización hemodinámica donde la prueba de clampeo permitió conocer la potencial repercusión del tiempo anhepático, y de esta manera volemizar en forma anticipada al paciente logrando disminuir la hipotensión posterior, asociado al uso balanceado de DVA.

Es destacable la baja mortalidad, menor al 10\% incluyendo cirugías piloto, y baja morbilidad, donde no tuvimos filtración de anastomosis ni complicaciones vasculares mayores en el seguimiento. Los fallecimientos se produjeron en el periodo de reperfusión, similar a otros modelos ${ }^{18,20}$. El tiempo quirúrgico total y el tiempo anhepático son comparables a lo descrito en la literatura internacional. La baja en la presión arterial y ph postreperfusión son esperables, y en la mayoría de los casos se logró recuperar en el seguimiento.

Una de nuestras limitaciones es el breve tiempo de seguimiento, lo cual fue un requerimiento impuesto por el comité de ética. Es un desafío en la siguiente etapa, con un modelo ya consolidado, poder prolongar este periodo. El hecho de incluir los pilotos, sin que esto fuera en desmedro de los resultados, se logró a través de la estandarización del procedimiento y el uso de modelos ya probados adaptándolos a la realidad local.

\section{Conclusión}

El presente trabajo resume la consolidación de un modelo de cirugía experimental en porcino para trasplante, y refleja el primer paso en investigación clínica de nuevas tecnologías asociadas al trasplante hepático en Chile.

\section{Agradecimientos}

$\mathrm{Al}$ aporte de cada uno de los miembros del equipo, que gracias a su dedicación y perseverancia lograron que se consolidará este proyecto. Cirujanos digestivos, becados de subespecialidad, cirugía general e investigación, anestesista veterinaria, enfermera coordinadora, alumna de medicina, arsenaleras, bioquímico y auxiliares.

\section{Responsabilidades éticas}

Protección de personas y animales. Los autores declaran que para esta investigación no se han realizado experimentos en seres humanos.

El proyecto fue aprobado por el Comité de Ética de Bienestar Animal de la Pontificia Universidad Católica de Chile con el acta de aprobación $\mathrm{n}^{\circ}$ 11160928 y cumple con normativa establecida por la comisión europea para investigación con animales.

Confidencialidad de los datos. L os a utores declaran que en este artículo no aparecen datos de pacientes.

Conflictos de interés: no hay.

\section{Bibliografía}

1. Martin P, DiMartini A, Feng S, Brown Jr, Fallon M, et al. Evaluation for liver transplantation in adults: 2013 practice guideline by the American Association for the Study of Liver Diseases and the American Society of Transplantation.
Hepatology 2014;59:1144-65.

2. Nacif L, Kim V Galvao F, Ono S, Pinheiro $\mathrm{R}$, Carrilho F, et al. Translational medical research and liver transplantation: systematic review. Transl Gastroenterol Hepatol. 2018;3:91.

3. Goodrich Jr, Welch H, Nelson J, Beecher T, Welch C. Homotransplantation of the canine liver. Surgery 1956;39:244-51.

4. Cordier G, Garnier H, Clot JP, Camplez $\mathrm{P}$, Gorin JP, Clot P, et al. Orthotopic liver graft in pigs. 1st results. Mem Acad Chir. (Paris) 1966;92:799-807.

5. Esmaeilzadeh M, Nickkolgh A, Majlesara A, Hafezi M, Garoussi C, GhaziMoghaddam K. Technical guidelines 
for porcine liver allo-transplantation: a review of literature. Ann Transplant. 2012;17:101-10.

6. Calne R, Yoffa D, White H, Maginn R. A technique of orthotopic liver transplantation in the pig. Br J Surg. 1968;55:203. https://doi.org/10.1002/ bjs. 1800550310.

7. Oldhafer K, Hauss J, Gubernatis G, Pichlmayr R, Spiegel H. Liver transplantation in pigs: A model for studying reperfusion injury. J Invest Surg. 1993;6:439-45.

8. Knubben K, Thiel C, Schenk M, Etspuler A, Schenk T, Morgalla M, et al. A New Surgical Model for Hepatectomy in Pigs. Eur Surg Res. 2008;40:41-6.

9. Nykonenk A, Vavra P, Zonca P. Anatomic Peculiarities of Pig and Human Liver. Experimental and Clinical Transplantation 2017;1:21-6.

10. Cooper DK, Satyananda V, Ekser B, van der Windt $D$, Hara $H$, Ezzelarab $\mathrm{MB}$, et al. Progress in pig-to-nonhuman primate transplantation models (1998-2013): a comprehensive review of the literature. Xenotransplantation 2014;21:397-419.
11. Cooper DK, Dou KF, Tao KS, Yang ZX, Tector AJ, Ekser B. Pig Liver Xenotransplantation: A Review of Progress Toward the Clinic. Transplantation 2016;100:2039-47.

12. Fondevilla C, Hessheimer AJ, Flores E, Vendrell M, Muñoz J, Escobar B, et al. Step-by-step guide for a simplified model of porcine orthotopic liver transplant. J Surg Res. 2011;167:e39-45.

13. Heuer M, Reinhardt R, Kneiseler G, Wurzinger P, Zou X, Hua F, et al. Liver Transplantation in Swine without Venovenous Bypass. Eur Surg Res. 2010;45:20-5.

14. Fung SK, Hui TW, Wong AK, Lei GM. Anaesthesia for liver transplantation: experience at a teaching hospital. HKMJ Vol 5 No 1 March 1999;5:27-33.

15. Kaiser G, Heuer M, Fruhauf N, Kuhne C, Broelsch C. General Handling and Anesthesia for Experimental Surgery in Pigs. Journal of Surgical Research 2006;130:73-9.

16. Gu J, Tao G, Liu D, Guo Y, Wang H, Lu K. Hemodynamic Monitoring in Pigs Undergoing Orthotopic Abdominal Multivisceral Transplantation.
Transplantation Proceedings 2009;41:4376-81.

17. Thiel K, Schenk M, Etspuler A, Schenk T, Morgalla M, Konigsrainer A, et al. A simple dummy liver assist device prolongs anhepatic survival in a porcine model of total hepatectomy by slight hypothermia. BMC Gastroenterol. 2011;11:79. Published online 2011 Jul 14. doi: 10.1186/1471-230X-11-79.

18. Yandza T, Tauc M, Saint-Paul M, Ouaissi M, Gugenheim J, Hétuberne X. The pig as a preclinical model for intestinal ischemia-reperfusion and transplantation studies. Journal of Surgical Research 2012;178:807-19.

19. Copca N, Hanna A, Pivniceru C, Constantinica V, Radilescu G, Gardean G, et al. Experimental Liver Transplantation on Pigs - Technical Considerations. Chirurgia 2013;4:542-6.

20. Canedo B, Galvao F, Ducatti L, Nacif L, Catanozi S, Soler W, et al. Liver Autotransplantation in Pigs without Venovenous Bypass: A Simplified Model using a Supraceliac Aorta CrossClamping Maneuver. Ann Transplant 2015;20:320-6. 